\title{
El género Cycloneda Crotch (Coleóptera: Coccinellidae) en el departamento del Cusco, Perú
}

\section{The genus Cycloneda Crotch (Coleoptera: Coccinellidae) in the department of Cusco, Peru}

\section{Abdhiel Bustamante-Navarrete}

Colección Entomológica de la Universidad Nacional San Antonio Abad del Cusco. Gabinete C338, Pabellón C, Ciudad Universitaria de Perayoc. Av. de la Cultura, 733, Cusco, Perú. E-mail: abdhie177@gmail.com

\section{Resumen}

El examen de especímenes de la familia Coccinellidae, depositados en la Colección Entomológica de la Universidad Nacional San Antonio Abad del Cusco, Perú, permitió determinar la presencia de siete especies del género Cycloneda Crotch, 1871: Cycloneda marcapatae Bustamante, Oroz \& Cosio 2009, Cycloneda vandenbergae González, Bustamante \& Oróz 2008, Cycloneda andresi Oróz, Bustamante \& Cosio 2009, Cycloneda dieguezi González 2018, Cycloneda arcula (Erichson 1847), Cycloneda ecuadorica (Timberlake 1943), y Cycloneda sanguinea (Linnaeus 1763). Los taxones encontrados se hallan presentes en 35 localidades, de 24 distritos, en 10 provincias del departamento del Cusco, ocupando un rango altitudinal que abarca desde los 800 msnm (Sahuayaco, Echarate, La Convención), hasta los 5,230 msnm (Pucacocha, Ausangate, Quispicanchi). Para cada especie se presenta una diagnosis, distribución, comentarios sobre su biología, fotografías de su habitus, y microfotografías del aparato genital.

Palabras clave. Coccinella, Coccinellina, Coccinellini, control biológico, diversidad, predación, taxonomía.

\begin{abstract}
The examination of specimens of the Coccinellidae family, deposited in the Entomological Collection of the National University San Antonio Abad del Cusco, Peru, allowed determining the presence of 7 species of the genus Cycloneda Crotch 1871: Cycloneda marcapatae Bustamante, Oroz \& Cosio 2009, Cycloneda vandenbergae González, Bustamante \& Oróz 2008, Cycloneda andresi Oroz, Bustamante \& Cosio 2009, Cycloneda dieguezi González 2018, Cycloneda arcula (Erichson 1847), Cycloneda ecuadorica (Timberlake 1943), and Cycloneda sanguinea (Linnaeus 1763). The species are present in 35 localities, of 24 districts, in 10 provinces of the department of Cusco, occupying an altitude range that ranges from 800 meters above sea level (Sahuayaco, Echarate, La Convención), to 5,230 meters above sea level (Pucacocha, Ausangate, Quispicanchi). For each species, a diagnosis, distribution, comments on its biology, photographs of its habitus, and photomicrographs of the genital tract are presented.
\end{abstract}

Keywords. biological control, Coccinella, Coccinellina, Coccinellini, diversity, predation, taxonomy. 


\section{Introducción}

El género Cycloneda Crotch, 1871, se halla presente en el Caribe, Norte, Centro, y Suramérica, y es, en esta última, donde la fauna parece ser más diversa, particularmente en las regiones montañosas, templadas y australes (González \& Vandenberg, 2006). Las especies del género parecen alimentarse principalmente de áfidos, y ocasionalmente en otros insectos y ácaros de cuerpo blando (Vandenberg, 2002).

El nombre Cycloneda fue usado por primera vez por Crotch (1871), como remplazo del nombre Daulis Mulsant, 1850, nombre que estaba pre ocupado por el género australiano Daulis Erichson, 1842 (Coleóptera: Endomychidae). El mismo autor, (Crotch, 1873), efectuó la revisión de los Coccinellidae de los Estados Unidos y, recién allí, elaboró la diagnosis del género Cycloneda, e indicó que este género era muy próximo a Coccinella Linnaeus, 1758 y Harmonia Mulsant, 1850; posteriormente, (Crotch, 1874), restringió este género al Nuevo Mundo, es por ello que muchas especies incluidas hoy en Cycloneda, anteriormente fueron consideradas por Timberlake (1943) dentro de Coccinellina Timberlake, género actualmente considerado como un sinónimo (Iablokov-Khnzoryan, 1990).

Gordon (1985) revisó las especies de Cycloneda de América del Norte y México, e indicó que este género contenía más de 50 especies en las regiones Neartica y Neotropical. El género fue estudiado nuevamente por Vandenberg (2002), quien lo redefinió con una visión menos restringida en torno a la especie tipo, incluyendo al género varias especies anteriormente ubicadas en
Coccinellina Timberlake, y Pseudoadonia Timberlake, pero también dejando fuera gran cantidad de especies, que aún están formalmente incluidas en Cycloneda, y que no corresponden a la definición dada por esta autora(González, 2018).

En el Perú, González Fuentes et al. (2008), y Oróz Ramos et al. (2009) describieron nuevas especies del género, elevando el número de taxones en el país a 10 (González, 2015), posteriormente González (2018) adicionó 4 taxones adicionales.

En el departamento del Cusco, las especies Coccinellina shannoni Timberlake, Coccinella petitti Mulsant, y Coccinellina sp. fueron citadas efectuando control biológico en distintos cultivos del departamento (Carrasco, 1962, 1968;Yábar \& Tisoc, 1988).

Distribución: Estados Unidos, México, Guatemala, Honduras, Nicarágua, Costa Rica, Panamá, Venezuela, Colombia, Ecuador, Perú, Bolívia, Chile, Brasil, Paraguay, Argentina y Uruguay(Araujo-Siqueira \& Almeida, 2006).

\section{Área de estudio}

El departamento del Cusco se ubica en la sierra sur del Perú, y limita con otros 7 departamentos, abarcando una extensión de 71 $986.50 \mathrm{~km} 2$. El medio físico del departamento es extremadamente variado, ya que confluyen alineamientos montañosos, nudos, altiplanos y mesetas, así como profundos valles y cañones, con un rango altitudinal comprendido entre los 250 msnm (distrito de Echarate, provincia de La Convención) y los 6,373 msnm (nevado Ausangate, provincia de Quispicanchi).

\section{Métodos}

Se examinaron especímenes de la familia Coccinellidae, presentes en la Colección 


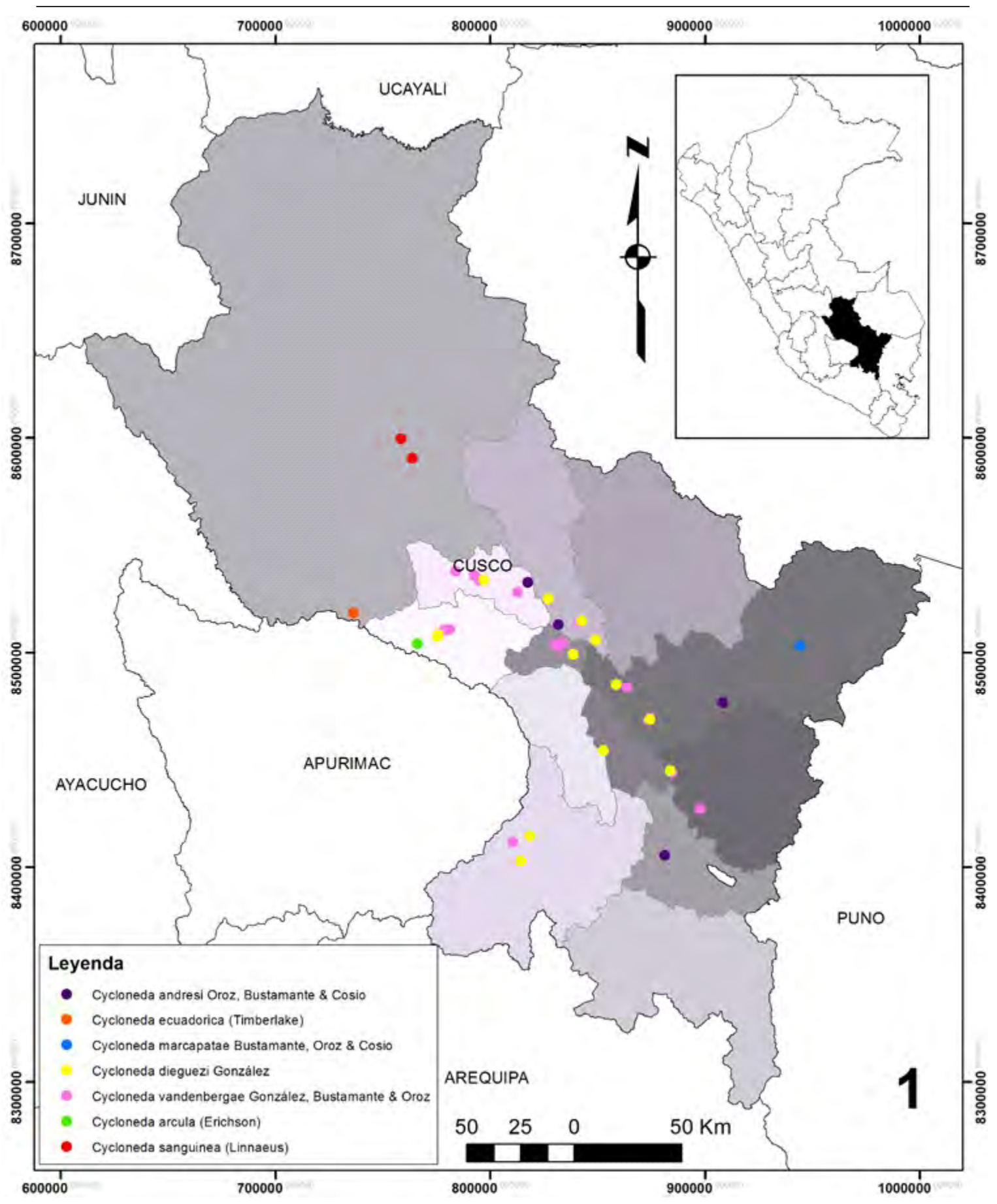

Figura 1. Localidades de colecta de las especies del género Cycloneda Crotch, 1871, en el departamento del Cusco. 
Entomológica de la UNSAAC (CEUCUNSAAC). Cada espécimen se hallaba montado en un alfiler entomológico, junto a una o más etiquetas que contienen los datos de colecta.

Para la identificación fue necesaria la extracción del aparato genital masculino, y en algunos casos el femenino. Para ello se separó el abdomen completo, que luego fue introducido en un vial con una solución de hidróxido de potasio al $10 \%$, y puesto en un vaso de precipitación con agua y sometido al calor durante 5-10 minutos. El abdomen luego fue lavado en alcohol y dispuesto en una placa portaobjeto con una gota de glicerina. Los aparatos genitales fueron extraidos, bajo observación en el microscopio estereoscopio, con ayuda de estiletes finos, y finalmente fueron conservados dentro de microviales con glicerina montados en los alfileres junto a su respectivo ejemplar.

El habitus de cada espécimen fue fotografiado con una cámara AxioCam ICc5 5 montada en un microscopio estereoscopio Discovery V20®; los aparatos genitales fueron fotografiados con una cámara Truechrome II $\mathbb{R}$ instalada en un microscopio Novel N-800m. En ambos casos, las fotografías fueron hechas en enfoque secuencial y combinadas usando el programa Helicon Focus $\AA$, y editadas en el programa Paint.net $\mathbb{R}$ para mejorar el brillo, contraste y para corregir imperfecciones.

La información geográfica de los ejemplares fue procesada en el programa Google Earth $\AA$, y luego exportada en formato kmz para luego ser importada al programa ArcGis $\mathbb{R} 10$, donde se transformó al formato shape (shp). Se usaron capas geográficas base de la Carta Nacional Digital (capa de departamentos y provincias) para la elaboración del mapa de localidades de colecta.
Para cada especie se presenta una diagnosis, distribución, comentarios sobre su biología, fotografías de su habitus, y microfotografías del aparato genital. Para la información del material examinado se transcribieron, literalmente, los datos de las etiquetas, e información faltante y/o relevante fue añadida entre corchetes .

Todos los especímenes estudiados se encuentran depositados en la Colección Entomológica de la Universidad Nacional San Antonio Abad del Cusco, Perú

\section{Resultados}

Se determinaron 7 especies del género Cycloneda Crotch, 1871: Cycloneda marcapatae Bustamante, Oroz \& Cosio 2009, Cycloneda vandenbergae González, Bustamante \& Oróz 2008, Cycloneda andresi Oroz, Bustamante \& Cosio 2009, Cycloneda dieguezi González 2018, Cycloneda arcula (Erichson 1847), Cycloneda ecuadorica (Timberlake 1943), Cycloneda sanguinea (Linnaeus 1763).

Los ejemplares provienen de 35 localidades de colecta, en 24 distritos (de un total de 112 en el departamento), de 10 provincias del departamento del Cusco (Figura 1). El rango altitudinal de las localidades de colecta está comprendido desde los 800 msnm (Sahuayaco, Echarate, La Convención), hasta los 5,230 msnm (Pucacocha, Ausangate, Quispicanchis).

\section{Taxonomía}

\section{Género Cycloneda Crotch, 1871}

Especie tipo: Coccinella sanguinea Linnaeus, 1763

El género se encuentra asimilado a un grupo de géneros definido por Vandenberg (1992) como "Cycloneda, Erythroneda y aliados", 
caracterizado por el aparato genital del macho con el penis comprimido antes del ápice, por la guía del penis no bifurcada en el ápice, generalmente cónica u ojival, y con el aparato genital de la hembra con un infundibulum esclerosado, cilíndrico o cónico y ensanchado en uno o ambos extremos (González, 2018).

\section{Cycloneda andresi Oroz, Bustamante \& Cosio 2009 (Figuras 4, 13, 14).}

Diagnosis: longitud: 3-4.2 mm. Cuerpo de forma oval. Presenta el pronoto negro con un borde lateral angosto de color blanco además de dos manchitas ovaladas pequeñas en el disco. Los élitros son anaranjados con una franja sutural negra ensanchada en la mitad anterior en forma de rombo y en el ápice además de dos manchas discales negras paralelas al margen lateral (Figura 4)

Distribución: PERÚ (Ancash, Cajamarca, Cusco, Huancavelica, Pasco) (González, 2007; Oróz Ramos et al., 2009). Localidad tipo: Ancash, Perú(Oróz Ramos et al., 2009).

Especie descrita sobre material proveniente de los departamentos Cusco, Ayacucho y Ancash, y que es muy próxima a Cycloneda ancoralis (Germar) que presenta un diseño elitral similar. Los ejemplares de la localidad de Pucacocha (laguna) fueron colectados en el piso ecológico de puna donde se alimentaban de pulgones dentro del musgo de la zona, compartiendo el hábitat con ejemplares de Eriopis andina Hofmann (Oróz Ramos et al., 2009).

Material examinado (Figura 1): (01 ej.) $\mathrm{C}$ h i c ón $3,170 \mathrm{~m} \mathrm{~s} \mathrm{n} \mathrm{m} \mathrm{,}$ Chicón/Urubamba/Cusco, 16.06.1992, E. Yábar col, Det. G. González 2012; (01 ej.) Koricocha 21.03.2008, Cusco/Cusco 4041 msnm, 13²6'33.58” S 71 55'59.27” O, W. Cosio L. col; (01 ej.) Pucacocha laguna, $\mathrm{C}$ ? / 03 / 0 \% 9 / 200007 , Ausangate/Ocongate/Cusco, 5,230 msnm,
19L 0254878 UTM 8471436, W. Cosio L. col; (01 ej.) Perú, Cusco, Canas, Quehue, Chirupampa, 19/II/2013, 3949 msnm, $14^{\circ} 21^{\prime} 30^{\prime \prime S ~} 71^{\circ} 26^{\prime} 56^{\prime}$ W, J.A. Cruz col.

\section{Cycloneda arcula (Erichson 1847) (Figuras 6, 17, 18).}

Diagnosis: longitud: $3.5 \mathrm{~mm}$. Forma ovalada. Cabeza negra con región interocular anaranjada. Pronoto negro con borde crema regular y angosto y dos manchitas claras en el disco en forma de ",". Élitros anaranjados con manchas negras variables, desde solo dos, la primera al centro del disco y la segunda alargada en el declive apical, paralela al borde lateral hasta ejemplares con élitros negros con el borde lateral y cinco manchas amarillas (Figura 6).

Distribución: CHILE, PERÚ (Ancash, Arequipa, Cajamarca, La Libertad, Lima) (González, 2007, 2018). Localidad tipo: Perú (Erichson, 1847).

Esta especie fue descrita como Coccinella arcula Erichson, 1847 con material procedente de Perú, posteriormente Mulsant (1850) describió a Coccinella petitti sobre la base de un morfo similar lo que causó que ambas sean sinonimizadas. Posteriormente el género Coccinella fue restringido a especies del viejo mundo y las especies americanas son reordenadas en el género Cycloneda (González, 2006). González (2018) revisó ejemplares de la especie procedentes de Ancash, Cajamarca, Arequipa, la Libertad y Lima, y describió por primera vez el aparato genital de ambos sexos, por otro lado estableció la extensión de las variaciones en su diseño elitral estableciendo dentro de esta especie a varios morfotipos poco pigmentados, especialmente en la parte norte de su distribución. 
Los ejemplares examinados en el presente estudio corresponden con las variaciones poco pigmentadas de la especie.

Material examinado (Figura 1): (02 ej.) Mollepata, 3,200 msnm, Anta, Cusco, agosto 2001, C.E.R. col.

\section{Cycloneda dieguezi González 2018 (Figuras 5, 15, 16).}

Diagnosis: longitud: 3.2-5 mm. Forma oval. Pronoto negro con un borde claro y dos manchas pequeñas en el disco. Élitros negros con un patrón de manchas amarillas o anaranjadas: tres manchas laterales unidas al borde y dos manchas suturales más grandes, una basal y la otra en los 3/4 del élitro (Figura 5).

Distribución: PERÚ: Apurímac, Ayacucho, Cusco (González, 2007, 2018). Localidad tipo: Ayacucho, Perú(González, 2018).

Carrasco (1962, 1968), y (Yábar \& Tisoc, 1988 ) reportaron a Coccinellina sp., alimentándose de áfidos en cultivos de maíz en las localidades de Perayoc, San Sebastián, Anta y en el Valle de Urubamba. La diagnosis de la especie, en ambos trabajos, corresponde a un morfo muy cercano a la especie Cycloneda arcula (Erichson), descrita para Perú como Coccinella arcula Erichson 1847. González (2018) revisó ejemplares procedentes de la provincia de Urubamba identificados como Coccinella arcula, coincidentes además con la descripción de Carrasco, y determinó que en realidad pertenecían a una nueva especie, la cual describió como Cycloneda dieguezi.

Material examinado (Figura 1): (01 ej.) Q u i quijan a $\quad 3400 \mathrm{~m}$, Quiquijana/ Quispicanchi / Cusco, 24/Febrero/2005, Quinua, J.F. Costa y J. Farfán F. col; (01 ej.) San Salvador 2,800, Pisac/ClCalca/Cusco, 06.02.2005, A.
-A. Bustamante col; (02 ej.) Checacupe, Checacupe/Canchis / Cusco, 15.02.1999, C.E.R. col; (01 ej.) Wañacahua 3,740 msnm, Sto. Santo Tomas / Chumbivilcas / Cusco, 05.02.1993, E. Yábar col; (02 ej.) Primavera, Ollanta/Urubamba / Cusco, 22.01.2001, C.E.R. col; (03 ej.) Misquiyacu 2785m, Limatambo/Anta/Cusco, 16.02.1993, E. Yábar col; ( $01 \mathrm{ej}$.$) Misquiyacu,$ Limatambo/Anta/Cusco, 22.01.1995, M. Delgado col; (01 ej.) Acos, Acos/01.2003, A. Meléndez col; (01 ej.) Esmeralda 3230 msnm, Sto.Santo Tomas / Chumbivilcas / Cusco, 17.01.1995, E.Yábar col; (01 ej.) PERÚ: CU. Kayra (INV), $13^{\circ} 84^{\prime} 00.00 ” S / 71^{\circ} 64^{\prime} 00.00 ” W$, 3,320m, 17.X.2013, E. Mondragón, T. Montoya col; ( 03 ej.) Pis a c, Pisac/ClCalca/Cusco, 08.01.1992, M. Lloclla col; ( $01 \mathrm{ej.)} \mathrm{Urco} \mathrm{2,800} \mathrm{msnm,}$ Urco/ClCalca/Cusco, 05.10.2002, J.F. Costa col; (04 ej.) sin datos de colecta, C.E.R. col; (01 ej.) Col. O. Ochoa M., Cusco, Tancarpata, 25-02-1993; (01 ej.) Salloc, Andahuaylillas, Quispicanchi, Cusco, julio 2017, Puya ferruginea, C.E.R. col.

\section{Cycloneda ecuadorica (Timberlake 1943) (Figuras 7, 19, 20).}

Diagnosis: longitud: 3.2-4.5 mm. Cuerpo de forma semicircular, alargada; el pronoto, negro, presenta un borde lateral claro, más ancho hacia la base, con dos manchas pequeñas, oblicuas en el disco. Los élitros anaranjados, con dos manchitas difusas claras en la base (Figura 7).

Distribución: ECUADOR, PERÚ (Ancash, Cusco, Junín, La Libertad, Tumbes) (González, 2007).

Timberlake (1943) describió esta especie dentro del género Coccinellina, género creado 
- para incluir a las especies neotropicales del género Coccinella. Posteriormente IablokovKhnzoryan (1990) considero a Coccinellina como sinónimo menor de Cycloneda Crotch, género restringido a la fauna del nuevo mundo (Araujo-Siqueira \& Almeida, 2006).

Externamente su apariencia es muy similar a Cycloneda sanguinea Mulsant, pero presenta un menor tamaño, un color más claro y las manchas pronotales más desarrolladas, además de que su aparato genital es muy diferente (González, 2007).

Recientemente registrada para el Perú, en el departamento de Ancash (González, 2015). Es el primer registro de la especie para el departamento del Cusco.

Material examinado (Figura 1): (12 ej.) PERÚ, Cusco, La Convención, Santa Teresa, Choquequirao, -13.3928-72.8642, diciembre 2017, 2,700 msnm, A. Laurel col; (09 ej.) PERÚ, Cusco, La Convención, Santa Teresa, Choquequirao, -13.3928 -72.8642, 2,700 msnm, A. Laurel col; (12 ej.) PERÚ: Cusco/Quillabamba, Sta. Teresa Choquequirao, -13.3928 -72.8642, 505 /V / 2018, 2700 m, A. Laurel col.

\section{Cycloneda marcapatae Bustamante, Oroz \& Cosio 2009 (Figuras 2, 9, 10)}

Diagnosis: longitud 3-4 mm. Cuerpo oval, convexo. Élitros negros con manchas amarillentas. El pronoto es negro con una banda blanco amarillenta contorneando los bordes frontal y lateral. Los élitros son negros, con tres manchas amarillentas en cada uno, ubicadas en cada tercio del élitro y una banda marginal que presenta un fuerte engrosamiento en la zona media del élitro (Figura 2).

Distribución: PERÚ (Cusco) (Oróz Ramos
(Oróz Ramos et al., 2009). Localidad tipo: Marcapata, Perú(Oróz Ramos et al., 2009).

Taxón descrito con base en ejemplares colectados en la localidad de Marcapata, provincia de Quispicanchi, dentro de vegetación mixta de pajonal (Poaceae) y Asteraceae, en una zona con agrupaciones de Chusquea sp. (Poaceae) (Oróz Ramos et al., 2009). El material tipo se encuentra depositado en la Colección Entomológica de la UNSAAC.

Material examinado (Figura 1): (05 ej.) PERÚ, Cusco, 3,500 msnm ¿?, Huayrackunca, Marcapata, Quispicanchi, 19L 289647 8497285, 15.IV.2008, 3,200msnm, W. Cosio L. \& A. Oroz R. colector.

\section{Cycloneda sanguinea (Linnaeus 1763) (Figuras 8, 21, 22).}

Diagnosis: longitud: 3-6 mm. Forma semicircular. Pronoto negro con un borde claro angosto además de dos manchas diagonales en el disco. Élitros anaranjados a rojos, sin manchas (Figura 8).

Distribución: Cosmopolita: ARGENTINA, BOLIVIA, CHILE, ECUADOR, URUGUAY, COLOMBIA, VENEZUELA, PANAMÁ, GUATEMALA, NICARAGUA, COSTA RICA, BELICE, MÉXICO, CUBA, USA, CANADÁ. Probablemente toda AMÉRICA, EL CARIBE, PERÚ (Ancash, Cusco, Ica, Junín, Lima, Loreto, Madre de Dios, Tumbes) (González, 2007).

Es una de las especies más características de Coccinellidae, y una de las más estudiadas, que pese a su poca variabilidad en el diseño, ha sido descrita varias veces(González, 2007).

Es citada como controlador biológico de la “mosca blanca lanuda” de los cítricos, Aleurothrixis floccosus, y de los pulgones de los 
cítricos, Aphis citricidus Kirby y Toxoptera aurantii (Beingolea, 1959a, 1961), además de ser una especie importante dentro de la entomofauna benéfica del cultivo del algodonero (Beingolea, 1959b; Gonzalez, 1962; Herrera, 1961; Lobaton, 1959; Martin, 1959, 1960; Piedra, 1960).

En el departamento del Cusco, este escarabajo ha sido colectado en diferentes localidades de la provincia de La Convención sobre cultivos de cítricos como naranjos y limoneros en donde se alimenta de pulgones del género Toxoptera (Carrasco, 1962, 1968).

Material examinado (Figura 1): (04 ej.) $\mathrm{L}$ i $\mathrm{m} \mathrm{a} \mathrm{t} \mathrm{a} \mathrm{m} \mathrm{b} \mathrm{o} 2500 \mathrm{~m} \mathrm{~s} \mathrm{n} \mathrm{m} \mathrm{,}$ Limatambo/Anta/Cusco, 12.08.2003, A. Bustamante col; (04 ej.) Sangobatea, Echarate/LC La Convención/Cusco, 03.03.1972, J. Escalante col; (03 ej.) Sahuayaco, marzo 1996, 800 msnm, R. Casafranca col; (03 ej.) Rumira, Cusco, 21.03.2000, C.E.R. col.

\section{Cycloneda vandenbergae González, Bustamante \& Oróz 2008 (Figuras 3,} 11, 12).

Diagnosis: longitud: 3.2-4.5 mm. Cuerpo de forma oval. Presenta manchas elitrales negras que forman tres franjas longitudinales: una sobre el disco de cada élitro y otra formada por la unión de los bordes sutúrales negros. Eventualmente las franjas discales pueden estar separadas por el centro formando dos manchas ovaladas cada una (Figura 3).

Distribución: PERÚ: Cusco (González Fuentes et al., 2008). Localidad tipo: Urubamba, Cusco, Perú (González Fuentes et al., 2008).

Carrasco $(1962,1968,1987)$ identificó a Coccinellina shannoni Timberlake y Coccinella petitti Mulsant alimentándose de pulgones del del maíz y kiwicha. Las descripciones proporcionadas y el material depositado por este autor en la Colección Entomológica de la UNSAAC, parecen indicar que Carrasco consideró como especies diferentes a dos variaciones de una misma especie, y que corresponderían en realidad a este taxón recientemente descrito, que se encuentra distribuido entre los 2,500-3,500 m de altitud, es bastante común en su área de distribución, y se encuentra principalmente sobre cultivos de maíz, alimentándose de Aphis maidis y permanecen hasta después de la época de cosecha(Carrasco, 1962).

Material examinado (Figura 1): (03 ej.) Kayra, 3,220 msnm, San Jerónimo/Cusco/Cusco, 10/Marzo/2005, Quinua, J.F. Costa y J. Farfán F. col; (01 ej.) Pumamarca 3450m, S.San Sebastián/Cusco/Cusco, 14.09.2002, J.F. Costa col; (01 ej.) Salineras 3330, San Sebastián/Cusco/Cusco, 31.01.2004, A. Bustamante col; (01 ej.) Phiry, $2470 \mathrm{msnm}$, Ollantaytambo / Urubamba / Cusco, 25/09/2004, Trat: 2, Y. Valdez - A. Alfaro col; $\left(\begin{array}{lll}0 & 1 & \text { e j . ) }\end{array}\right.$ P hi r y, $2470 \mathrm{~m}$, Ollantaytambo/ Urubamba / Cusco, 15/01/2005, Trat: 2, Y. Valdez - A. Alfaro col; ( 02 e j.) Phiry, $2800 \mathrm{msnm}$, Ollantaytambo/ Urubamba / Cusco, 25.11.2004, maíz, A. Bustamante col; (04 ej.) Rumira, Cusco, 21.03.2000, C.E.R. col; (02 e j.) Prim a vera, O l l a n t a Ollantaytambo/Urubamba / Cusco, 22.01.2001, C.E.R. col; (01 ej.) Urubamba 2,877msnm, Urubamba/Urubamba/Cusco, 15/Noviembre/2003, B2T3, A. Alfaro - Y. Valdez col; (02 ej.) Pisaq, Pisaq/Calca/Cusco, 27/Enero/2005, Quinua, J.F. Costa J. Farfán F. col; (01 ej.) Pisac, Pisac/ClCalca/Cusco, 08.01.1992, M. Lloclla col; (02 ej.) Pisac, Pisac / ClCalca / Cusco, Diciembre-Febrero 2005, C.E.R.col; (03 ej.) Misquiyacu- 

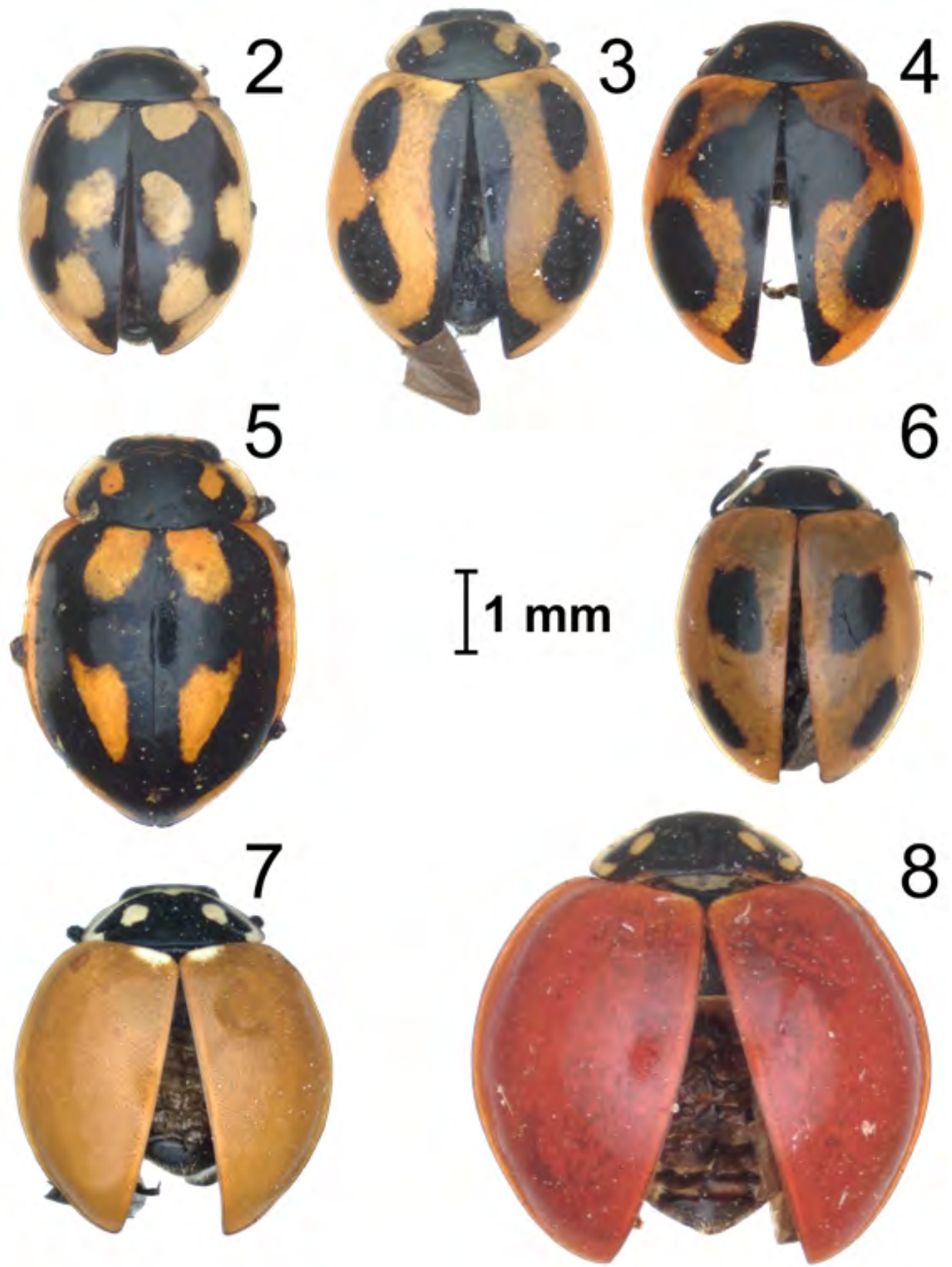

Figura 2. Cycloneda marcapatae Bustamante, Oroz \& Cosio; figura 3. Cycloneda vandenbergae González, Bustamante \& Oróz; figura 4. Cycloneda andresi Oroz, Bustamante \& Cosio; figura 5. Cycloneda dieguezi González 2018; figura 6. Cycloneda arcula (Erichson); figura 7. Cycloneda ecuadorica (Timberlake); figura 8. Cycloneda sanguinea (Linnaeus). 


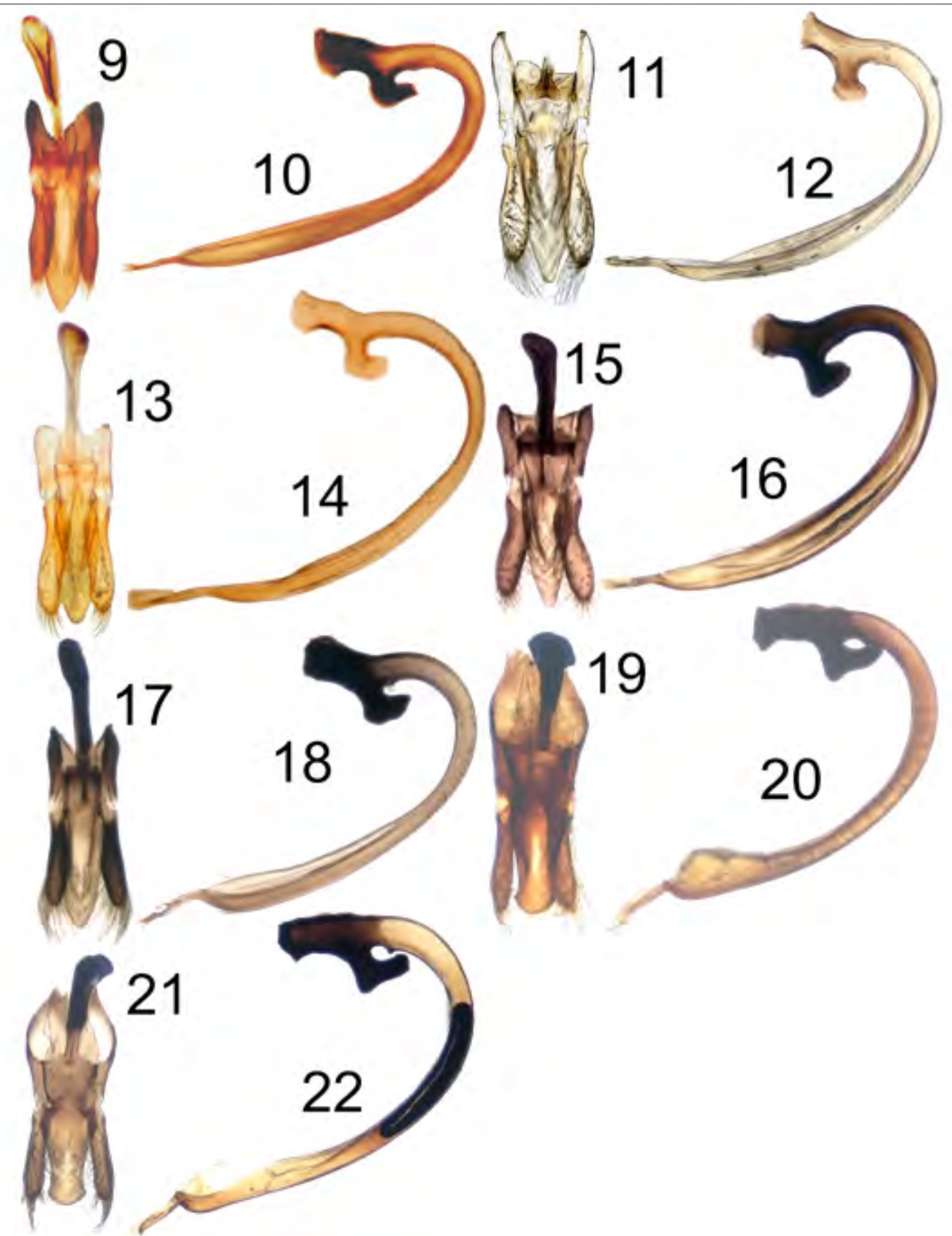

Figura 9 - 10. Cycloneda marcapatae Bustamante, Oroz \& Cosio: figura 9. tegmen, figura 10. penis. Figuras 11-12. Cycloneda vandenbergae González, Bustamante \& Oróz: figura 11. tegmen, figura 12. penis. Figuras 13-14. Cycloneda andresi Oroz, Bustamante \& Cosio: figura 13. tegmen, figura 14. penis. Figuras 15-16. Cycloneda dieguezi González 2018: figura 15. tegmen, figura 16. penis. Figuras 17-18. Cycloneda arcula (Erichson): figura 17. tegmen, figura 18. penis. Figuras 19-20. Cycloneda ecuadorica (Timberlake): figura 19. tegmen, figura 20. penis. Figuras 21-22. Cycloneda sanguínea (Linnaeus): figura 21. Tegmen y figura 22. penis. 
2,785 msnm, Limatambo/Anta/Cusco, 16.02.1993, E. Yábar ( $\mathrm{col}) ;(01 \mathrm{ej.})$ Limatambo 2500, Limatambo/Anta/Cusco, 12.12.1966, C.E.R. col; (01 ej.) Huayllojo $3450 \mathrm{~m}$, Checacupe / Canchis / Cusco, 04.08.1994, E.Yábar col; (01 ej.) Llusco 3,480 $\mathrm{m} \mathrm{s} \mathrm{n} \mathrm{m,} \quad \mathrm{S} \mathrm{t} \mathrm{o} \quad \mathrm{S}$ a n t o Tomas / Chumbivilcas/Cusco, 16.06.1993, E. Yábar col; (01 ej.) Quiquijana 3,400m, Quiquijana/Quispicanchi / Cusco, 24/Febrero/2005, J.F. Costa y J. Farfán F. col; ( 01 e j.) H uaro $3235 \mathrm{~m} \mathrm{snm}$, Urcos/Quispicanchi/Cusco, 03.12.1992, E. Yábar col; (02 ej.) Salineras 3,330, San Sebastián/Cusco/Cusco, 31.01.2004, A. Bustamante col; (01 ej.) Salineras 3,350, San Sebastián/Cusco/Cusco, 31.01.2004, A. Bustamante col; (01 ej.) Aparquilla 3,330m, Anta/Cusco, 12.01.1993, E.Yábar col; (01 ej.) $\begin{array}{llllllllllll}\mathrm{P} & \mathrm{r} & \mathrm{i} & \mathrm{s} & \mathrm{c} & \mathrm{a} & \mathrm{c} & \mathrm{u} & \mathrm{c} & \mathrm{h} & \mathrm{o} & \end{array}$ Ollantaytambo/Urubamba / Cusco, 22.12.2000, C.E.R. col; (05 ej.) Quiquijana, 3400m, Quiquijana/Quispicanchi/Cusco, 24/Febrero/2005, Quinua, J.F. Costa y J. Farfán F. col; (02 ej.) Col. O. Ochoa M.,YucayCusco, 21.11.1992. I. Tisoc; (01 ej.) Col. O. Ochoa M., Urubamba, 03-03-1988, maíz, I. Tisoc; (1 ej.) Perú, Cusco, Canchis, San Pablo, Chara, -14.241199 -71.316154, 28/x/2017, 3, 579 msnm,Y. Mamani - G. Choque.

\section{Discusión}

De las 16 especies citadas en el país, siete (el $43 \%)$ se encuentran en el departamento, haciendo del Cusco, la localidad con mayor diversidad del género. A esto hay que añadir que la especie Cycloneda reclusa Weise, 1902, también es citada para el departamento, en la localidad de Marcapata (Weise, 1902), aunque desde su descripción no ha vuelto a ser colectada.
Las citas previas, en el departamento del Cusco, de las especies Coccinellina shannoni Timberlake, Coccinella petitti Mulsant, Coccinella sp., y Coccinellina sp. corresponden en realidad a otros taxones, algunos de reciente descripción, pertenecientes al género Cycloneda. Esto demuestra la importancia de los trabajos de revisión y actualización de la entomofauna, así como la conformación de colecciones para el depósito y resguardo de material biológico. Es necesario destacar que de las siete especies determinadas en el presente estudio, cuatro fueron recientemente descritas, y de ellas tres provienen de material biológico procedente del departamento.

El registro preliminar de las especies muestra a Cycloneda vandenbergae, Cycloneda dieguezi, y C. andresi con la distribución más amplia del género en el departamento (en 7, 8, y 4 provincias respectivamente). En tanto las especies C. arcula, C. marcapatae, y C.ecuadorica, tienen una distribución más restringida (1 provincia). Es necesario aclarar que la mayoría de las colectas fueron efectuadas cerca a campos de cultivo, y que existe un gran vacío de información en localidades de las zonas de selva de las provincias de La Convención, Calca, Paucartambo y Quispicanchi.

En base a las altitudes, Cycloneda sanguinea $(800$ msnm-2,850 msnm), y C. ecuadorica (2,700 $\mathrm{msnm})$ se presentan en altitudes relativamente propias de valles interandinos, donde Cycloneda dieguezi (2,785 msnm-3,740 msnm) también tiene presencia, aunque esta especie además alcanza ambientes altoandinos, en los cuales $C$. arcula, y C. marcapatae (3,200 $\mathrm{msnm}$ ) parecen tener una distribución restringida a este piso. En tanto Cycloneda andresi (3170 msnm-5230 msnm) está habituada a altitudes más altas, llegando al borde nival.

\section{Agradecimientos}

Al Dr. Erick Yábar Landa, por la utilización de los equipos de fotografía y microfotografía del Laboratorio de Entomología de la E.P. de Biología. 


\section{Literatura citada}

Araujo-S iqueira, M., \& Almeida, L. (2006). Estudo das espécies brasileiras de Cycloneda Crotch (Coleoptera, Coccinellidae). Revista Brasileira de Zoologia, 23(2), 550-568.

Beingolea, O. (1959a). El problema de la "Mosca Blanca Lanuda" de los cítricos, en el Perú Aleurothrixus floccosus (Homop.: Aleurodidae). Revista Peruana de Entomología, 2(1), 65-68.

Beingolea, O. (1959b). Notas sobre la Bionómica de Arañas e Insectos Benéficos que ocurren en el Cultivo del Algodón. Revista Peruana de Entomología, 2(1), 36-44.

Beingolea, O. (1961). El Valle de Palpa como ejemplo de las posibilidades de integrar medidas de control químico y biológico en las plagas de los arboles cítricos. Revista Peruana de Entomología, 4(1), 1-3.

Bicho, C., \& Almeida, L. (1998). Revisao do genero Neocalvia Crotch (Coleoptera, Coccinellidae). Revista Brasileira de Zoologia, 15(1), 167-189. DOI: 10.1590/S010181751998000100015

Carrasco, F. (1962). Coccinellidos predatores en la Región del Cuzco. Revista Peruana de Entomología, 5(1), 91-93.

Carrasco, F. (1968). Lista preliminar de insectos del departamento de Entomología. Revista de La Facultad de Ciencias, Universidad Nacional de San Antonio Abad Del Cusco, 177-191.

Carrasco, F. (1987). Insectos en la "kiwicha" cultivada en Cusco y Apurímac. Revista Peruana de Entomología, 30(1), 38-41.

Crotch, G. (1871). List of the Coccinellidae. Cambridge, 8p.

Crotch, G. (1873). Revision of the Coccinellidae of the United States. Transactions of the American Entomological Society, Philadelphia, 45, 363-382.

Crotch G. 1874. A revision of the Coleopterous Family Coccinellidae, University Press, London, $311 \mathrm{p}$.

Erichson,W. F. (1847). Conspectus insectorum coleopterorum quae in Republica peruana observata sunt. Archiv Für Naturgeschichte., 13, 67-185.

González Fuentes, G., Bustamante Navarrete, A., \& Oróz Ramos, A. (2008). Aporte al conocimiento del género Cycloneda Mulsant (Coleoptera: Coccinellidae): nuevas especies, de Chile y Perú. Boletín de la Sociedad Entomológica Aragonesa, 43(1), 81-87.

González, G. (2006). Los Coccinellidae de Chile en línea, recuperado el 15 de setiembre del 2020 . http://www.coccinellidae.cl/paginasWebChile/Paginas/Inicio Chi.php

González, G. (2007). Los Coccinellidae de Perú en línea, recuperado el $15 \mathrm{de} \quad \mathrm{se} t \mathrm{i}$ e m b r e d e l 2010. https://www.coccinellidae.cl/paginasWebPeru/Paginas/Inicio Peru.php

González, G. (2014). Coccinellidae. En S. Roig-Juñent, L.

Claps, \& J. Morrone (Eds.), Biodiversidad de Artrópodos Argentinos

(Vol. 3, pp. 509-530). Editorial INSUE-UNT.

González, G. (2015). Beetles (Coleoptera) of Peru: A Survey of the Families. Coccinellidae. Journal of the Kansas Entomological Society, 88(2), 229-236. DOI: 10.2317/kent-88-02-229-236.1

González, G. (2018). A porte al conocimiento de la tribu Coccinellini (Coleoptera: Coccinellidae) en América del Sur. Revista Chilena de Entomología, 44(2), 169-206.

González, G., \& Vandenberg, N. (2006). Review of lady beetles in the Cycloneda germainii species complex (Coleoptera; Coccinellidae: Coccinellinae: Coccinellini) with descriptions of new and unusual species from Chile and surrounding countries. Zootaxa, 1311,13-50. DOI: 10.11646/zootaxa.1311.1.2

González, P. M. (1962). Plagas del algodonero en elValle de Tambo e irrigación. Revista Peruana de Entomología, 5(1), 84-90.
Gordon, R. (1985). The Coccinellidae (Coleoptera) of America North of Mexico. Journal of the New York Entomological Society, 93(1), 352-599.

Herrera, J. M. (1961). Problemas entomológicos en el cultivo de los algodones Tanguis y Pima en el Perú. Medidas de control y su organización. Revista Peruana de Entomología, 4(1), 58-66.

Hofmann, W. (1970). Die Gattung Eriopis Mulsant (Col. Coccinellidae). Mitteilungen Der Münchner Entomologischen Gesellschaft (e.V.), 60, 102-116.

Iablokov-Khnzoryan, S. (1990). About the Classification of the Coccinellini. Coccinella, 2, 58-60.

Lobatón, M. (1959). Equilibrio biológico de Mescinia peruella Schaus en la zona baja del Valle de Pisco, Campaña 1958-59. Revista Peruana de Entomología, 2(1), 113-115.

Martin, H. (1959). Algunos datos sobre parásitos y predatores del Bucculatrix en el Valle del Chira. Revista Peruana de Entomología, 2(1), 110-111.

Martin, H. (1960). Estudios sobre biología, hábitos de vida, ecología y control de Bucculatrix en el departamento de Piura. Revista Peruana de Entomología, 3(1), 46-53.

Mulsant, E. (1850). Species des coléoptères trimères sécuripalpes. In Annales des Sciences Physiques et naturelles, d'agriculture et d'industrie, ser. 2, 2 (1104 p.). Maison.

Oróz Ramos, A., Bustamante Navarrete, A., \& Cosio Loaiza, W. (2009). Aporte al conocimiento del género Cycloneda Crotch (Coleoptera: Coccinellidae): nuevas especies del Perú. Boletín de la Sociedad Entomológica Aragonesa, 45(1), 293-297.

Piedra, V. (1960). Problemas entomológicos actuales del Valle de Chira. Revista Peruana de Entomología, 3(1), 36 40.

Timberlake, P. (1943). The Coccinellidae or Lady beetles of the Koebele Collections, part I Hawaii. Bulletin of the Experimental Station of the Hawaiian Sugar Planters'Association Entomological, 22, 1-67.

Vandenberg, N. (1992). Revision of the New World Lady Beetles of the Genus Olla and Description of a New Allied Genus (Coleoptera: Coccinellidae). Annals of the Entomological Society of America, 85(4), 370-392.

Vandenberg, N. (2002). The new world genus Cycloneda Crotch (Coleoptera: Coccinellidae): Historical review, new diagnosis, new generic and specific synonyms, and an improved key to North American species. Proceedings of the Entomological Society of Washington, 104(1), 221-236.

Vandenberg, N., \& Gordon, R. (1988). The Coccinellidae (Coleoptera) of South America: PART I. A revision of the genus Erythroneda Timberlake 1943. Revista Brasileira de Entomología, 32(1), 31-43.

Vandenberg, N., \& Gordon, R. (1996). A new genus of neotropical Coccinellini (Coleoptera: Coccinellidae) related to Olla Casey and allies. Proceedings of the Entomological Society of Washington, 98(3), 541-550.

Yábar, E., \& Tisoc, I. (1988). Artrópodos predatores asociados al maíz en el Valle Urubamba, Cusco. Revista Peruana de Entomología, 31(1), 143-146.

Weise J. (1902). Coccinelliden aus Süd Amerika III. Deutsche Entomologische Zeitschrift 1902: 161-176. 